\title{
Telemedicina: mejora de la calidad en la atención de los pacientes críticos desde la fase prehospitalaria hasta el servicio de medicina intensiva
}

\author{
G. Murias ${ }^{\mathrm{a}}$, B. Sales ${ }^{\mathrm{b}}$, O. García-Esquirol ${ }^{\mathrm{c}}$ y L. Blanch ${ }^{\mathrm{d}, *}$
}

a Unidad de Cuidados Intensivos, Clínica Bazterrica y Clínica Santa Isabel, La Plata, Buenos Aires, Argentina

bInstitut Universitari Fundació Parc Taulí, Corporació Parc Taulí, Universitat Autónoma de Barcelona, Sabadell, España

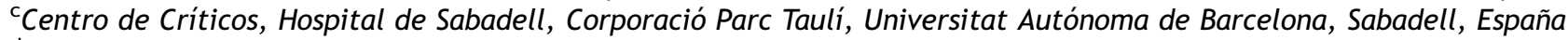

${ }^{d}$ Centro de Críticos, CIBER Enfermedades Respiratorias (CIBERes), Hospital de Sabadell, Corporació Parc Taulí, Institut

Universitari Fundació Parc Taulí, Universitat Autónoma de Barcelona, Sabadell, España

Recibido el 16 de enero de 2009; aceptado el 9 de mayo de 2009

Disponible en Internet el 6 de octubre de 2009

\section{PALABRAS CLAVE \\ Telemedicina; \\ Pacientes críticos; \\ Costes sanitarios; \\ Alarmas avanzadas}

\begin{abstract}
Resumen
El sistema asistencial sanitario está en crisis, y los cuidados críticos (desde los sistemas de transporte hasta la unidad de cuidados intensivos [UCI]) no se libran de esta circunstancia. La falta de integración entre la Medicina prehospitalaria y los hospitales receptores, una limitación profunda en el número de especialistas en cuidados críticos y unos recursos económicos que no aumentan a la par del incremento en la demanda de cuidados intensivos son las piedras angulares del problema. Es más, las previsiones de futuro no son alentadoras.

Varios estudios demuestran que las UCl “cerradas”, en las que un especialista en cuidados intensivos dirige la atención del paciente, tienen mejores resultados que las $\mathrm{UCI}$ "abiertas", en las que la atención sigue a cargo de los médicos de atención primaria. Sin embargo, que un especialista en cuidados intensivos se encuentre al lado del paciente es cada vez más complicado; sólo un cambio en la manera de trabajar podría ayudar a incrementar el número de pacientes cuidados por un intensivista. La tecnología de la información y la capacidad de comunicación (transmisión de datos en línea) deberían explotarse al máximo para aumentar tanto la cobertura como la calidad de los cuidados intensivos. Lejos de ser un reemplazo del modelo existente, la telemedicina podría ser la herramienta complementaria que ayudara a mejorar la manera en la que los médicos intensivistas atienden a sus pacientes.

(c) 2009 Elsevier España, S.L. y SEMICYUC. Todos los derechos reservados.
\end{abstract}

\footnotetext{
*Autor para correspondencia.

Correo electrónico: Iblanch@tauli.cat (L. Blanch).
} 


\section{KEYWORDS}

Telemedicine;

Critically ill patients;

Healthcare costs;

Advanced alarms
Telemedicine: Improving the quality of care for critical patients from the pre-hospital phase to the intensive care unit

\begin{abstract}
The Health System is in crisis and critical care (from transport systems to the ICU) cannot escape from that. Lack of integration between ambulances and reference Hospitals, a deep shortage of critical care specialists and assigned economical resources that increase less than critical care demand are the cornerstones of the problem. Moreover, the analysis of the situation anticipated that the problem will be worse in the future.

"Closed" ICUs in which critical care specialists direct patient care outperform "open" ones in which primary admitting physicians direct patient care in consultation with critical care specialists. However, the current paradigm in which a critical care specialist is close to the patient is in the edge of the trouble so, only a new paradigm could help to increase the number of patients under intensivist care. Current information technology and networking capabilities should be fully exploited to improve both the extent and quality of intensivist coverage. Far to be a replacement of the existing model Telemedicine might be a complimentary tool. In fact, to centralize medical data into servers has many additional advantages that could even improve the way in which critical care physicians take care of their patients under the traditional system.
\end{abstract}

(c) 2009 Elsevier España, S.L. and SEMICYUC. All rights reserved.

\section{Introducción}

El centro coordinador recibe una llamada de urgencia. Un cuestionario de no más de 3 o 4 preguntas (dirigidas por las respuestas) le permite caracterizar la situación y enviar el recurso apropiado más cercano o de menor tiempo de respuesta. Al llegar al lugar, el equipo asistencial encuentra a un paciente con dolor precordial indicativo de isquemia miocárdica y decide iniciar el tratamiento y el traslado. Una vez en la unidad de soporte vital avanzado, monitoriza al paciente e inicia una videoconferencia con el médico del Centro Coordinador del Servicio de Atención a Emergencias Médicas (SAEM) (nombre ficticio). Éste dispone de sonido e imagen de la cabina de la ambulancia y de las señales biológicas del monitor en tiempo real. De esta manera, ayuda a tomar decisiones al disponer de información similar a la que tendría de manera presencial (ve al paciente y hasta podría interrogarlo, observa los trazados del monitor y del ventilador si lo precisara). La unidad se dirige a un hospital con servicio de hemodinámica para realizar angioplastia primaria. El médico a cargo del servicio de medicina intensiva (unidad de cuidados intensivos [UCI]) acepta al paciente y se hace corresponsable de su atención junto con el equipo asistencial del SAEM, porque participa en la videoconferencia con la ambulancia y el Centro Coordinador. Las señales biomédicas que recibe del paciente quedan almacenadas de manera inmediata en la historia clínica digitalizada de éste. El diagnóstico es claro y con la unidad aún de camino llama a los médicos del servicio de hemodinámica para que vayan al hospital. El paciente llega a la sala de hemodinámica y tras el procedimiento, se traslada a la $\mathrm{UCl}$, donde se monitoriza enviando sus registros a continuación de los ya almacenados durante el traslado.

Lamentablemente, este escenario dista mucho del real. El sistema de salud está colapsado en general y los costes se han incrementado (atención más costosa y mayor demanda) sin que se incrementen de manera paralela los recursos asignados a éste. En Medicina Intensiva este problema se agrava, porque aun si los recursos económicos estuvieran disponibles, una limitación marcada y creciente del recurso humano capacitado funcionaría como una limitante adicional.

\section{Problemas durante la atención prehospitalaria}

Las emergencias médicas constituyen un problema en aumento. Desde el conocimiento por parte de la población de la existencia de servicios de atención a las emergencias como vía rápida de asistencia en el hospital, las llamadas de urgencia han crecido un $8 \%$ anual (un 30\% más que las urgencias). Esto ha hecho que las falsas llamadas de emergencia, casi inexistentes a mediados de la década pasada, representen actualmente cerca del $20 \%$ del total (1,1 millones de falsas emergencias sobre 5,6 millones de llamadas por año) ${ }^{1}$.

Los servicios de urgencias constituyen el principal mecanismo de acceso de la población a las instituciones asistenciales. Si se excluyen los ingresos generados por nacimientos, el $55 \%$ de las admisiones hospitalarias se producen desde los servicios de urgencias ${ }^{2}$. Esto hace evidente la importancia de una adecuada gestión de éstas y la trascendencia sanitaria de las medidas que la mejoren.

En este escenario podría esperarse que los recursos asignados al sistema se hubieran incrementado para acompañar el incremento de las urgencias. Lamentablemente, esto no ha sido así. De hecho, en ocasiones, las limitaciones presupuestarias han hecho, incluso, que éstos se recorten ${ }^{3-7}$.

Existen también problemas organizativos. Los equipos de atención prehospitalaria están pobremente integrados con los servicios de urgencias hospitalarios. Esto redunda en una deficiente utilización de los recursos disponibles y demoras 
en el tratamiento médico, con las consecuencias que ello conlleva.

El impacto de los retrasos en la atención de víctimas de trauma se conoce desde hace casi un siglo. El análisis de la mortalidad de los soldados franceses de la Primera Guerra Mundial mostró que cuando se reanimaba a las víctimas dentro de la primera hora, moría el $10 \%$, mientras que la cifra se elevaba al $75 \%$ cuando el retraso era de $8 \mathrm{~h}^{8}$. Estos datos parecen haber sido el soporte para la postulación por el Dr. Adams Cowley del concepto de “hora de oro". Aun cuando una ventana de tiempo en particular no parece tener ningún sustento bibliográfico ${ }^{8}$, es claro que las demoras en la reanimación tienen un profundo impacto sobre la calidad de los resultados. Con el correr del tiempo, se hizo evidente que una atención rápida no sólo era crítica en las víctimas de trauma, sino en las urgencias cardiovasculares ${ }^{10}$, en las urgencias neurológicas $^{11}$ y en la sepsis ${ }^{12}$.

En parte teniendo en cuenta lo anterior, en junio de 2006 después de 3 años de trabajo, una comisión designada por el Institute of Medicine de los Estados Unidos presentó los resultados de una investigación sobre el futuro de las urgencias médicas $^{9-11}$. Sus principales recomendaciones podrían resumirse en 4 puntos:

1. Integración de las diferentes etapas en la atención del paciente. La atención de las urgencias médicas desde la etapa prehospitalaria hasta su destino final en el hospital debe estar integrada.

2. Derivación adecuada. No todos los hospitales resultarán adecuados para un paciente individual en la medida que las especializaciones y los servicios de los primeros, al igual que las necesidades de los últimos, son altamente variables. Un sistema que funcione correctamente debería derivar a cada paciente al lugar más adecuado para atender su enfermedad.

3. Garantía de mejores prácticas disponibles de acuerdo con el conocimiento del momento. En cada etapa de atención, el paciente debe recibir el mejor tratamiento disponible. Esto tiene 2 alcances principales: no sólo hay que garantizar qué se hace, sino cuándo se hace.

4. Posibilidad de evaluación de los resultados del sistema. El funcionamiento de un sistema podrá considerarse adecuado sólo si es capaz de demostrar que sus resultados están dentro de unos estándares de calidad esperados. Para ello, es necesario planear el sistema de manera que sus resultados puedan fácilmente medirse y compararse. Idealmente, debería contar con herramientas para determinar la causa de los errores y malfuncionamientos para permitir corregirlos en el futuro.

El lugar y el momento en el que ocurrirá una urgencia no pueden preverse. Dado que el tiempo para la implementación del tratamiento influencia los resultados, el sistema debe estar optimizado para agilizar la respuesta y enviar lo antes posible una unidad con complejidad adecuada para resolver el problema. Una vez en el sitio de la urgencia, el mejor tratamiento debería instaurarse en forma inmediata. En algunas ocasiones, el equipo del SAEM resuelve el problema sin necesidad de asistencia. En otras, sin embargo, es un médico del centro coordinador el que de forma remota debe tomar las decisiones, guiado por escasa información oral que recibe por vía telefónica o por radio. Si se traslada, será necesario avisar al personal que recibirá al paciente para que prepare los recursos necesarios para su atención (la información también será escasa). En la práctica, suele ser un administrativo del Centro Coordinado del SAEM el que informa al médico receptor sobre el estado del paciente.

Un sistema de telemedicina dirigido a asistir a los pacientes críticos durante su atención inicial y traslado proveería de herramientas al equipo del SAEM para tratar adecuadamente al enfermo $y$, en caso de necesidad, informar del centro más apropiado para su asistencia. En el hospital que acepta al paciente, el médico receptor debería tener la posibilidad de tomar decisiones sobre su tratamiento desde el mismo momento en el que acepta la derivación; tratamiento que será más eficiente cuanto más información se disponga del paciente. Esto tendría 2 consecuencias:

1. reducción del tiempo de inicio de un tratamiento adecuado,

2. y mejor preparación de los recursos para recibir al paciente.

\section{Situación en España}

Probablemente no pueda hablarse de una situación en España. En el mundo, en general, el sistema considera estrategias óptimas diferentes en las grandes ciudades (donde los centros de referencia suelen encontrarse a menos de $10 \mathrm{~min}$ de cualquier lugar) y en el ámbito rural (donde la norma es que los tiempos de traslado desde el lugar de un incidente a un centro de referencia superen los $15 \mathrm{~min})$. A esta fuente de heterogeneidad, se agrega en España que el tratamiento del problema es diferente en cada comunidad. Algunas decisiones locales, de hecho, parecen basarse en ideas diametralmente opuestas: casi a la par que los vehículos de actuación rápida se reemplazan en Cataluña por sistemas de traslado, en Madrid aumentan su número e importancia en el sistema. Como contrapartida, al menos en algunas comunidades, los recursos asignados al sistema no han acompañado la tendencia mundial y, lejos de reducirse, se han incrementado. Como ejemplo, el número de unidades de soporte vital básico (que cuentan con técnico sanitario y enfermero) se incrementó en los últimos años un $44 \%$ en la ciudad de Barcelona y cerca de un $80 \%$ en el resto de Cataluña. Esto ha hecho que el sistema cuente en la actualidad con 352 unidades de soporte vital básico, 66 unidades de soporte vital avanzado (incluidos 4 helicópteros medicalizados) y 3 vehículos de actuaciones especiales.

\section{Problemas en cuidados intensivos intrahospitalarios}

A diferencia del resto de los pacientes, el paciente crítico requiere monitorización, pruebas complementarias, procedimientos y tratamiento de forma continua durante los 365 días del año. Esto representa unas necesidades muy altas de recursos humanos capacitados, que en muchas ocasiones el sistema no es capaz de cubrir, y es subóptimo el tratamiento de estos pacientes. Un estudio en Canadá reveló que cerca 
del $50 \%$ de las unidades no eran atendidas por especialistas y un $25 \%$ adicional contaba con ellos unas pocas horas por día. De hecho, el $90 \%$ de las unidades encuestadas en el estudio operaba fuera de los estándares de personal recomendados por la Society of Critical Care Medicine de los Estados Unidos. Aun cuando en la actualidad el problema en España es de menor magnitud, es posible anticipar que su importancia será mayor en el futuro. Al tiempo que el número de camas de cuidados intensivos ha ido en aumento (en 2008 era de 3.500 camas), las plazas de médicos internos y residentes (MIR) de medicina intensiva, principal determinante del número de nuevos especialistas en el futuro inmediato, han permanecido virtualmente inalteradas (416 entre 1996 y 1999; 412 entre 2000 y 2003, y 439 entre 2004 y $2007)^{12}$. Considerado en mayor profundidad, el análisis del problema se torna más complejo: el saldo migratorio de licenciados en Medicina y de especialistas es positivo y constituye, además, una tendencia en aumento. Entre los años 2003 y 2004, se homologaron un total de 2.611 títulos de licenciatura y 288 títulos de especialista, mientras que entre 2007 y 2008, estas cifras subieron a 5.383 y a 702, respectivamente ${ }^{13}$. Aun cuando sea difícil en este marco evaluar la situación actual, una búsqueda de ofertas laborales en la web en 2006 encontró 43 solicitudes de médicos intensivistas para sustituciones de larga duración o contratos de guardia, algunas de éstas no exigían MIR (síntoma de una especialidad deficitaria) $^{12}$.

El déficit de especialistas impide en muchas unidades que un intensivista se encuentre en presencia física, y el problema se ve agravado con el incremento progresivo de la demanda de camas de críticos (mejor tratamiento de pacientes politraumáticos, mayor número de cirugías complejas, etc.). Esta situación genera la creación de UCI de diferentes niveles, con diferentes posibilidades de brindar cuidados a sus pacientes. Las UCl de mayor complejidad suelen estar en centros urbanos con más recursos, mientras que extensas áreas geográficas más periféricas se encuentran descubiertas ${ }^{14-17}$. Cuando un paciente grave llega a una unidad con recursos asistenciales limitados, se intenta la derivación a otra unidad de mayor complejidad, aunque no es siempre posible. La inestabilidad hemodinámica, por ejemplo, implicaría unos riesgos no asumibles e impediría el traslado. La combinación de una elevada gravedad clínica y recursos asistenciales limitados supone para estos pacientes un pronóstico sombrío.

\section{Problema de los costes}

Cuando se analizan los costes en la sanidad, hay 2 premisas por destacar:

- representa un porcentaje muy elevado del producto bruto interno (PBI) en cualquiera de los sistemas estudiados,

- y el coste de la salud está aumentando a un ritmo cada vez mayor.

El coste de la salud en Estados Unidos en el año 2007 se incrementó un 6,9\% con respecto al año previo (el doble de lo que hubiera podido predecirse por la inflación) y llegó a
2,3 trillones de dólares, una cifra que representa el 16\% del $\mathrm{PBI}$.

Pero aun cuando el sistema en Estados Unidos parezca marcadamente ineficiente (los Estados Unidos gastan en la administración de los recursos de salud 6 veces más dinero per cápita que los países de Europa occidental ${ }^{18}$ ), la situación es también grave en otros países desarrollados. El coste de salud representa en Suiza el 10,9\% del PBI, en Alemania el $10,7 \%$, en Canadá el $9,7 \%$ y en Francia el $9,5 \%{ }^{19}$.

Una cama de cuidados intensivos cuesta 8 veces más que una cama de cuidados generales ${ }^{20}$ y el $64 \%$ de esos costes se atribuyen al coste del personal ${ }^{21}$. Considerando estos puntos, se hace evidente que con el paradigma actual no es la falta de especialistas el único motivo por el que el personal de las $\mathrm{UCl}$ no puede adecuarse a las recomendaciones internacionales; aun cuando los especialistas estuvieran disponibles, probablemente el sistema no podría pagarlos.

Tanto la atención como la monitorización de los pacientes críticos ingresados en las $\mathrm{UCl}(\mathrm{UCl}$, quirófano, urgencias, etc.) han seguido un modelo en el que el especialista se encuentra idealmente en el mismo lugar que el paciente. Debe encontrarse próximo al paciente para realizar prácticas y procedimientos, monitorizar la evolución de éste, acceder a la historia clínica, ver las imágenes y, finalmente, tomar las decisiones. Si bien para colocar accesos venosos centrales e intubar a pacientes no hay aún modelos alternativos y es, por tanto, mandatario que el encargado del procedimiento se encuentre cercano al paciente, no hay ninguna restricción para que el resto de las tareas pueda realizarse a distancia. Más aún, considerando que el nivel de capacitación y el tipo de entrenamiento necesario para realizar una y otra cosa son notoriamente diferentes, no hay ningún motivo por el que deba ser la misma persona la que realice ambas tareas. En España, un grupo de trabajo definió un conjunto de 146 competencias requeridas para la atención de pacientes críticos y las dividió en 2 niveles según hiciera falta una formación médica general (nivel uno) o una formación específica en cuidados intensivos (nivel 2). Las últimas representaron sólo un tercio del total $^{22}$.

De esta forma, la carencia de especialistas ha dado lugar a un modelo alternativo. En él, un enfermero/a o un médico en formación se encuentran físicamente con el paciente, mientras que el especialista solamente colabora en aquellos casos que se compliquen y requieran de su participación. Este modelo se puede implementar en forma única o complementaria del sistema tradicional (usualmente para cubrir horarios nocturnos, fines de semana y festivos). Si bien representa una buena alternativa, tiene por lo menos 2 consideraciones por resaltar:

1. El personal que se encuentra con el paciente debe detectar la existencia de un problema para solicitar asistencia. Muchas veces, ésta es una de las tareas que requieren mayor entrenamiento.

2. El especialista consultado debe tomar decisiones con fuentes de información claramente restringidas. El personal menos entrenado valora elementos como la forma de la curva de presión en la vía aérea, la variación de la presión del pulso, el perfil del capnograma, el 
patrón ventilatorio, los estudios de imágenes, el ECG, etc. y los transmite por vía telefónica. Esto conlleva el riesgo de pérdida de información y el de una interpretación inadecuada.

Con la telemedicina se pueden solventar ambos problemas. El agregado de inteligencia (mediante procesos automáticos y alarmas inteligentes) podría disparar la monitorización aun cuando el personal menos entrenado no hubiera sido capaz de detectar el problema. El segundo problema se solucionaría modificando la naturaleza del vínculo entre paciente y especialista. Al reemplazar la conexión de voz por una conexión de datos, puede transmitirse a distancia toda la información necesaria para hacer que la toma de decisiones sea más parecida a la del modelo presencial.

\section{Ventajas asociadas al uso de la telemedicina}

Además de las ventajas directas enunciadas, el uso de la telemedicina tiene otras ventajas asociadas. Es capaz de transmitir los datos provenientes de los dispositivos de monitorización y almacenarlos en la historia clínica digitalizada del paciente. Además, permite analizar los registros para mejorar nuestras acciones en asistencias futuras y aprender de nuestros errores y de nuestros éxitos.

\section{Sistemas de alarma más sofisticados}

El 77\% de los ingresos en la UCI se hace para monitorización (aun cuando finalmente sólo el 10\% requiera intervenciones mayores) ${ }^{23}$. Para ello, las $\mathrm{UCl}$ cuentan con sistemas que registran las variables fisiológicas de interés. Buscan minimizar situaciones de riesgo para el paciente mediante la implementación de una serie de alarmas que alertan al personal asistencial cuando éstos ocurren. Los fabricantes de estos sistemas de monitorización preocupados por evitar los falsos negativos (que un episodio verdadero no sea detectado) bajan el umbral de activación de las alarmas y generan un problema de sobreinformación. Kestin et al demostraron en su estudio que en quirófano, donde los pacientes suelen estar sedados y relajados, sólo el $3 \%$ de las alarmas alertaba sobre un episodio que representaba un riesgo real para el paciente ${ }^{24}$. La situación probablemente sea peor en las $\mathrm{UCl}$, donde la manipulación frecuente del paciente provoca falsas detecciones. Estas "falsas detecciones" comportan una serie de consecuencias:

1. La frecuencia de falsas alarmas es probablemente una de las causas por las que las alarmas se atienden con demora (o no se atienden en absoluto). En 2002, la Joint Commission on Accreditation of Health Care Organizations de los Estados Unidos revisó 23 casos de muerte o lesiones graves asociadas a ventilación mecánica y encontró que en el $65 \%$ de los casos el problema estuvo relacionado con retrasos en la atención de alarmas o fallo en la activación de alarmas debido a parámetros de configuración inadecuados (ningún caso de malfuncionamiento del equipo se detectó) ${ }^{25}$. Otro estudio demostraba que el personal llegaba a tolerar las alarmas hasta 10 min sin realizar intervenciones ${ }^{26}$.
2. El nivel de ruido al que los pacientes y el personal están expuestos en las $\mathrm{UCl}$ superó los $45 \mathrm{~dB}$ recomendados por la Environmental Protection Agency de los Estados Unidos, tanto durante el día como durante la noche, y llegó a detectar picos de $90 \mathrm{~dB}^{27}$. A este hecho se asoció la pobre calidad del sueño de los pacientes en la $\mathrm{UCl}^{28}$ y el burn-out del equipo de enfermería ${ }^{29}$.

La incorporación a los sistemas de monitorización de algoritmos más complejos podría mejorar sensiblemente el problema planteado por los sistemas de alarmas actuales. Hasta la fecha, las señales se han tratado como si no estuvieran relacionadas unas con otras. Aun cuando la señal del ECG y la pletismografía respiratoria son medidas mediante los mismos sensores, el mal funcionamiento de uno de los electrodos dispara usualmente una alarma de bajo nivel en el ECG ("electrodo desconectado") y una de máximo nivel en el pletismógrafo ("apnea”). La comprensión de los fenómenos fisiológicos y fisiopatológicos requiere una visión más global: el conjunto de bradicardia, hipertensión y aumento de la presión intracraneal brinda mucha más información que la suma de sus partes. Si bien cada uno de los elementos puede responder a múltiples causas, la combinación de las 3 alteraciones reduce drásticamente el nivel de incertidumbre y apunta al reflejo de Cushing como causa. La adquisición de señales por un sistema avanzado de telemedicina permitiría aumentar el nivel de integración mediante la incorporación de información proveniente de más de un dispositivo.

En ocasiones da más información en la variación de un parámetro vital en el tiempo que en su valor aislado. Una frecuencia cardíaca de $90 \mathrm{lpm}$ puede ser absolutamente normal, pero si una hora antes hubiera sido de $60 \mathrm{lpm}$, las consideraciones podrían ser otras. Aun así, persistirían problemas relacionados con la carga del personal dedicado a atender las alarmas y con la capacitación de éste, necesaria para solucionar los problemas que las alarmas evidenciaran.

Adquirida la información desde los equipos de monitorización, las alarmas no tendrían por qué limitarse a ser alertas sonoras emitidas en el box del paciente 0 , finalmente, en la central de monitorización. El sistema de telemedicina, mediante el uso de diversas puertas de enlace (gateways), podría emitir alarmas, como correos electrónicos, bípers, SMS, etc. Así, no sólo podría alertarse a distancia a un especialista de un episodio potencialmente peligroso, sino que se podría plantear un sistema de alarmas escalables en las que, de no solucionarse el problema después de un tiempo determinado de la activación de una alarma local, la alarma pudiera emitirse, por ejemplo, como un SMS a un supervisor.

\section{Recomendaciones de especialista}

El objetivo último de la programación de una alarma es mejorar la seguridad de los pacientes. En un medio en el que el recurso humano cualificado es escaso, el simple hecho de alertar sobre un episodio potencialmente peligroso no garantiza que este objetivo se cumpla. Así, la incorporación de información médica brindada por especialistas en los sistemas de monitorización podría ayudar a entender los 
fenómenos e indicar soluciones para los problemas. El nivel de integración que se logre y la calidad de la estructuración de la información de los registros médicos determinarán la utilidad de la información que pueda brindarse, garantizaría así la seguridad del paciente y daría una solución al problema de la detección de episodios importantes.

\section{Análisis automático de curvas}

Los sistemas de monitorización actuales muestran ondas y valores. Aun cuando las alarmas se emiten basadas en los últimos, el análisis de las primeras puede brindar muchísima información. Los especialistas suelen hacer uso de ellas mediante la inspección a pie de cama del paciente. Ejemplos son la aparición de una onda P2 mayor que P1 en el trazado de presión intracraneal que puede alertar sobre la reducción de la compliancia intracraneal, un flujo persistente en la vía aérea al final de la espiración que alertaría sobre la presencia de presión positiva intrínseca al final de la espiración (auto-PEEP), una variabilidad de la presión del pulso muy marcada que indicaría la necesidad de una carga de fluidos intravenosos, etc. Sin embargo, éste es un punto que requiere mayor entrenamiento. La implementación de procesos automáticos destinados a evaluar estos fenómenos es relativamente sencilla una vez que un sistema de telemedicina ha adquirido las señales de los dispositivos y la información está disponible independientemente del fabricante del monitor o del ventilador.

\section{Asistencia en los procesos de aprendizaje}

El proceso de formación tiene 2 componentes: una parte teórica, que puede ser presencial o mediante sistemas de e-Learning, y una parte de adquisición de capacidades y destrezas, que hasta hoy ha de ser necesariamente presencial.

Se espera del equipo humano en el ámbito de la salud un bajo nivel de errores en función de las consecuencias potenciales de éstos. Para esto, es necesario un elevado nivel de entrenamiento. El sistema de aprendizaje basado en casos (formación de especialistas, por ejemplo) genera los más altos niveles de capacitación. Este sistema es, sin embargo, laborioso y consume enormes cantidades de recursos y tiempo. Es aquí donde el e-Learning puede ser de mayor ayuda. Mediante simulaciones por ordenador, pueden recrearse los procesos intelectuales que llevan al aprendizaje, basado en casos reales previamente registra$\operatorname{dos}^{30}$.

A fines de la década pasada, Brandon Hall, editor de e-Learning Magazine, predijo que el $50 \%$ de la capacitación en el año 2003 se realizaría bajo alguna modalidad de entrenamiento electrónico. Para ese año, según la International Data Corporation, sólo en los Estados Unidos el negocio del e-Learning representó más de 11.000 millones de dólares, y marcó un crecimiento anual sostenido en el quinquenio de aproximadamente el $83 \%$ anual. Si bien el sector corporativo es el principal impulsor de ese crecimiento, el incremento de la potencia y la reducción del coste de los ordenadores, acompañados del desarrollo de más y mejores alternativas de software específico, han comenzado a acercar estas herramientas al ámbito académico.

Dentro de la esfera de lo que habitualmente se conoce como e-Learning, existen estrategias diferentes con muy diversos niveles de desarrollo tecnológico. En un extremo del espectro, el e-Learning no es más que la entrega de documentos electrónicos generados por aplicaciones de oficina (documentos de texto, planillas electrónicas, presentaciones de diapositivas, etc.). En este enfoque, los ordenadores sólo cambian la forma en la que los contenidos generados llegan a los alumnos, pero las características del proceso de aprendizaje permanecen inalteradas. En el otro extremo, sofisticados sistemas de simulación permiten cambiar las reglas del proceso y facilitan el aprendizaje basado en casos. Lamentablemente, la mayor parte de los sistemas de simulación disponibles encuentran como limitación que sólo está disponible un número acotado de casos previstos en la etapa de programación del sistema.

Un sistema de telemedicina podría integrarse con los sistemas de e-Learning con notables beneficios. El sistema permitiría guardar casos testigos y construir una biblioteca que pudiera utilizarse con fines docentes. Los casos en esta biblioteca podrían incluir la información del problema y las soluciones ensayadas de manera que permitiera el análisis posterior de lo actuado y el efecto de las maniobras o los cambios operados. Así, en lugar de simulaciones, esta solución permitiría el aprendizaje basado en casos reales y el análisis de los errores cometidos. Los casos podrían enriquecerse adjuntando a los registros notas, comentarios, resúmenes de historia clínica, capas de audio con mensajes con recomendaciones de especialistas, etc. (en la medida que estos elementos pueden agregarse como capas superpuestas, los registros originales no son modificados $)^{31}$.

Toda la información sobre el uso del material (visualización, comentario, invitaciones, preguntas, etc.) podría quedar registrada y lista para exportarse a cualquier sistema de e-Learning.

\section{Control de calidad}

Como se dijo, un sistema podrá considerarse adecuado si sus resultados pueden monitorizarse y compararse. Este es un motivo de preocupación cada vez más frecuente: un grupo de trabajo de la Sociedad Española de Medicina Intensiva, Crítica y Unidades Coronarias (SEMICYUC) definió recientemente 120 indicadores de calidad, 20 de los cuales se caratularon como de mayor importancia ${ }^{32}$.

Usualmente el control de calidad se realiza de manera diferida para la monitorización del sistema. La implementación de un sistema de telemedicina permite establecer alarmas de procesos. Así, el mismo sistema monitoriza en forma automática elementos relacionados con la calidad de su funcionamiento. Más allá de las ventajas que implica un control automático, una alerta sobre una violación de un protocolo podría disparar también acciones correctoras. De esta manera, el control de calidad mejoraría la calidad y lo sacaría del ámbito descriptivo. Una aproximación relativamente nueva a la gestión de la calidad de los sistemas de atención de pacientes críticos es la denuncia de los "casi errores" ${ }^{33}$. A diferencia de los efectos adversos, en los que un error o una complicación del proceso 
de atención generó un daño al paciente, en los "casi errores" una corrección oportuna logró evitar el daño. La ventaja que los "casi errores" tienen sobre los efectos adversos radica en que son igual de efectivos para detectar los puntos débiles de un proceso, pero al no haberse generado daño, el personal tratante los denuncia con mucha mayor facilidad. Nuevamente, un sistema de telemedicina puede diseñarse de manera que detecte estos episodios mediante procesos automáticos, y el almacenamiento de los registros puede aumentar el valor docente de éstos. Más allá de las implicaciones para el paciente individual, pueden diseñarse informes que monitoricen la calidad de trabajo en la $\mathrm{UCl}$, una carencia usual de los sistemas actuales ${ }^{34}$.

\section{Futuro}

Un gerente de una cadena de supermercados decide incrementar la rentabilidad de la empresa al favorecer en cada producto la venta de la marca comercial que le brinda mayor rentabilidad. Consulta a un especialista de marketing, que le explica que los productos afines tienden, cuando están próximos, a favorecer cada uno la venta del otro. Como ejemplo, le indica que asocie en el mismo exhibidor la cerveza y los snacks que le brindan mayor rentabilidad. El especialista conoce una regla "las personas que consumen cerveza usualmente consumen snacks". Ésta es la aproximación de un sistema experto: propone una solución basada en una regla conocida.

El conjunto de reglas con un grado de generalidad razonable que pueden enunciarse en situaciones complejas es, en general, limitado. En el ámbito de la salud, esto ha confinado a los sistemas expertos a problemas muy específicos (como el análisis de los valores del examen fisicoquímico y citológico de una muestra de líquido cefalorraquídeo (LCR) en un paciente con sospecha de meningitis).

Una aproximación diferente (profusamente explotada en el ámbito comercial y virtualmente desconocida en el campo de la salud) es la minería de datos. Al hacer uso de la ventaja que representa tener grandes bases de datos, el gerente analiza los recibos correspondientes a las ventas de años, y al aplicar técnicas de minería de datos, establece que los compradores de cerveza usualmente consumen peras. No conoce los motivos (la minería de datos no es explicativa), pero enuncia una nueva regla.

La formulación de hipótesis en el método científico ha seguido el modelo del sistema experto. El conocimiento de los procesos fisiológicos y fisiopatológicos lleva al investigador a enunciar una hipótesis (una regla) por testarse. Una vez testada, la hipótesis se transforma en conocimiento y forma parte del conjunto de información que se utilizará en la formulación de nuevas hipótesis.

Una ventaja adicional del uso de sistemas de telemedicina que adquieran información desde los equipos de monitorización es que permitirán crear enormes bases de datos de señales que podrían explotarse con técnicas de minería de datos. Imaginemos esto aplicado al ya enunciado problema actual de las alarmas. Veremos que el uso de técnicas de minería de datos, antes que contraponerse, puede ser complementario del uso de los sistemas expertos.
Reconsideremos el ejemplo presentado en el que la desconexión de un electrodo de ECG muestra una alarma de bajo nivel ("electrodo desconectado") en el ECG, mientras que en la frecuencia respiratoria la alarma es prioritaria ("apnea”). Un sistema experto muy básico podría decidir no disparar tal alarma mediante la aplicación de una regla sencilla, como "cuando un electrodo está desconectado, avisar la situación y suprimir la alarma de apnea”. Pero más allá de estas reglas obvias, es probable que pudieran encontrarse muchas otras.

Los equipos de monitorización conectados a un sistema de telemedicina capaz de adquirir y procesar los datos provenientes de éstos estarían conectados a un servidor local que ejecuta un sistema experto que, al valerse inicialmente de un conjunto acotado de reglas, decidiría cuándo alertar y cuándo no sobre la ocurrencia de un determinado episodio. Al mismo tiempo, los servidores de las diferentes $\mathrm{UCl}$ transmitirían a un servidor central capaz de construir una gran base de datos sobre la que se aplican técnicas de minería de datos. Al analizar las señales en su conjunto, se encontraría cada vez un número mayor de reglas (algunas obvias y otras inexplicables) y con ellas se alimentaría a los servidores locales que pasarían a tener un conjunto de reglas cada vez más rico y útil. Así, la sensibilidad de las alarmas podría incrementarse de manera discreta, al tiempo que la especificidad lo haría de manera exponencial. Al avanzar un paso más, cada vez que se diagnosticara un episodio potencialmente grave, como por ejemplo un episodio de fibrilación auricular, el sistema analizaría los registros correspondientes a la hora previa y podría encontrar un patrón repetitivo de alteraciones (poco frecuente en situaciones en las que el episodio no aparece). Si así fuera, se plantearían alarmas de cosas por suceder ("cuidado, es probable que su paciente presente una fibrilación auricular en el término de los próximos 60 min"). Esto representaría un cambio sustancial en lo que respecta a las alarmas: ya no sería el objetivo reducir los tiempos de respuesta ante un episodio, sino que los episodios podrían prevenirse.

\section{Requerimientos técnicos}

Se define el canal de comunicación como el medio utilizado para la transmisión de información o datos entre 2 puntos. Algunas de las características del canal de comunicación determinan las funcionalidades que pueden esperarse de un sistema de telemedicina.

El ancho de banda del canal es la capacidad o la cantidad de datos por unidad de tiempo que el medio es teóricamente capaz de transmitir. Debido a que el ancho de banda disponible no es ilimitado, cualquier aplicación que transmita información entre 2 puntos debe diseñarse teniendo en cuenta estas limitaciones. El sistema ideal es aquél escalable y adaptable a la capacidad de los diferentes canales sobre los que el sistema funcione o, lo que es lo mismo, aquél capaz de aprovechar la capacidad máxima del medio usado para la transmisión de la información. En España ${ }^{35}$, y en estos términos, cabe distinguir:

1. Redes fijas. Actualmente, y hasta 2010, será habitual disponer de un ancho de banda entre 3 y $20 \mathrm{Mbps}$. En el 
medio plazo (2010-2015) y a causa de la tecnología VDSL2 (Very-High-Bit-Rate Digital Subscriber Line 2) o las combinaciones de fibra óptica y VDSL, esta cifra se incrementará hasta los $50 \mathrm{Mbps}$. A partir del 2015, será habitual contar con redes de hasta $100 \mathrm{Mbps}$ a causa del uso de la fibra óptica hasta el propio domicilio o las combinaciones de fibra óptica y VDSL2.

2. Redes móviles. Los anchos de banda disponibles hasta 2010 estarán en torno a los 2 Mbps a causa de las redes de móviles $3 \mathrm{G}$ evolucionadas, aunque actualmente el mayor reto consiste en la generalización de este tipo de redes, que cubren apenas el 30\% del territorio. El ancho de banda se incrementará hasta los $15 \mathrm{Mbps}$ en el medio y el largo plazo (2010-2015) a causa de las tecnologías 3,5G vinculadas a tecnologías HSPA (High-Speed Packet Access). A partir de 2015 se podrán alcanzar anchos de banda en telefonía móvil de hasta 50 Mbps a partir de la llegada de las redes $4 G$, con tecnologías que están todavía en fase de investigación. En ambos casos, la tecnología de comunicaciones permite ya en la actualidad el desarrollo y la implementación satisfactorios de sistemas para la telemedicina.

El flujo de datos es la continuidad o la fluidez en la transmisión durante el tiempo que dura la comunicación. El flujo mide el rendimiento real del canal y depende del ancho de banda y la demora en la entrega de la información que en la práctica el medio puede garantizar. El flujo de datos resulta una medida incluso más importante que la del ancho de banda, puesto que en sistemas que transmiten información en tiempo real, en los que su constante actualización resulta crítica, el flujo es el que realmente determina la aptitud o la idoneidad del canal. Mientras que en redes fijas el flujo de datos está generalmente limitado con unas cotas mínimas y máximas, en redes móviles el problema es mayor debido a que resulta prácticamente imposible garantizar un flujo de datos mínimo por aspectos como la cobertura o la congestión de la red. Es por esto que los sistemas que funcionen sobre redes móviles deben diseñarse considerando las consecuencias que las características de este medio impone, como las oscilaciones en el flujo del canal e incluso la pérdida de conexión.

Al tratarse de datos sensibles, los sistemas de telemedicina tienen que operar, además, sobre canales que puedan garantizar la seguridad y la privacidad de la información. Existen diversos métodos para mantener la seguridad en un sistema y, entre otros, cabría mencionar las redes privadas virtuales, las técnicas de cifrado o el uso de certificados y firmas digitales.

En cualquier sistema para la práctica de la telemedicina es tan importante la información transmitida como lo son su calidad, su representación y su fiabilidad. En estos sistemas críticos, todos aquellos datos transmitidos que sean cuestionables en los términos previamente mencionados no sólo no tienen ningún valor, sino que además pueden inducir al error médico, con los riesgos que esto implica. El canal, por una parte, y el sistema usado, por otra parte, serán esenciales para garantizar la validez de la información en estos términos.

Toda vez que se transmitan ondas y no sólo valores aislados resulta clave identificar la mínima información que define el perfil y las características de las curvas. Por las características de los canales de comunicación actuales (con las correspondientes restricciones en cuanto a capacidad y a flujo de datos) resulta imprescindible determinar el mínimo volumen de datos requerido para una representación con calidad de investigación, completa e inequívoca de las ondas transmitidas.

Respecto a la presentación de la información, es igualmente importante facilitar la lectura de estas curvas. Es deseable que la interfaz de un sistema de telemedicina, la nomenclatura de las ondas y la forma de presentación sean homogéneas $\mathrm{y}$, por tanto, independientes del dispositivo que las está produciendo. En estos casos, es deseable que el sistema se encargue de la homogeneización de estas señales así como de su procesamiento — su interpolación y el ajuste de los diferentes relojes - para una presentación clara, totalmente sincronizada y el $100 \%$ ajustada a la morfología de las curvas originales. En el mismo sentido de mejorar la legibilidad y facilitar la comprensión de la información, es deseable la integración de fuentes diversas bajo una misma interfaz. Esto puede lograrse construyendo sistemas capaces de dar cuenta de todas las tareas o, lo que es más frecuente, puede lograrse garantizando la comunicación entre diferentes sistemas encargados de solucionar problemas puntuales. Para esto último es necesario que se establezcan estándares de comunicación y de almacenamiento de la información. Aun cuando su implementación sea claramente incompleta, el Health Leven 7 es un conjunto de estándares para el intercambio electrónico de información médica que probablemente se transforme en el estándar. Al englobar información clínica y administrativa, su especificación más utilizada es un estándar de mensajería para el intercambio electrónico de datos en salud, entre sistemas y subsistemas, por una parte, y entre éstos y el Sistema de Información Hospitalaria (HIS), por otra parte. Esto permite, por ejemplo, asignar unas pruebas o unos resultados concretos a un paciente concreto, bien en el HIS o bien en el sistema que corresponda. El estándar de almacenamiento está aún menos definido, con excepción del formato DICOM (Digital Imaging and Communication in Medicine) ampliamente utilizado para imágenes. A pesar de que fue inicialmente ideado para la gestión, el almacenamiento, la impresión y la transmisión de imágenes médicas, sus definiciones se han expandido a otros tipos de datos, como pueden ser las ondas fisiológicas (DICOM waveform). Teniendo en cuenta el enorme nivel de penetración que tiene y la cantidad de visualizadores disponibles para este formato, es probable que en el futuro inmediato pase a ser el estándar de almacenamiento.

Además de facilitar su comprensión, la captura sincronizada y con la suficiente definición de todas estas señales, es un requisito imprescindible para que éstas puedan utilizarse en términos de investigación, docencia, sistemas expertos y en la aplicación de técnicas de minería de datos.

\section{Conclusiones}

El uso de la telemedicina tiene ventajas obvias: permite brindar atención de especialistas a pacientes que han demostrado beneficiarse de ella en situaciones en las que el recurso humano no esté disponible o el sistema no pueda 
pagarlo. Sin embargo, estas enormes ventajas directas podrían incluso sobrepasarse debido a las ventajas indirectas relacionadas con la posibilidad de adquirir, normalizar, sincronizar y almacenar señales médicas. En un futuro inmediato, podrían estar disponibles para ampliar los alcances de los sistemas de alarmas, mejorar la especificidad de éstos y permitir el disparo de alarmas cualitativamente diferentes, esto es, no sólo alertas sonoras emitidas al lado de la cama del paciente, sino correos electrónicos, SMS, pagers enviados en primera instancia o como un sistema de escalamiento en un recorrido predefinido cuando los problemas no sean solucionados de forma satisfactoria. En lo inmediato, podrían contribuir no ya a cambiar la manera en la que los conocimientos médicos se aplican, sino también a modificar la manera en la que el conocimiento en sí mismo se produce. El uso de técnicas de minería de datos sobre grandes bases de datos de señales médicas podría producir enormes cantidades de información y constituirse en una nueva estrategia para formular hipótesis por testarse mediante la aplicación del método científico. Más allá de esto permitiría examinar las señales en formas que son imposibles para el ojo desnudo, como el análisis de éstas en dominios diferentes del temporal, tal vez el más notorio, el de las frecuencias. Es razonable incluso esperar que estos análisis revelen alteraciones sistemáticas en alguna o varias señales antes de la aparición de un episodio. Esto plantea la posibilidad de emitir alertas no ya sobre un episodio que ha ocurrido, sino sobre uno que puede ocurrir.

\section{Bibliografía}

1. Health and Social Care Information Centre (UK). Ambulance services, England: 2004-05. United Kingdom: Departament of Health (UK); 2005.

2. Owens PEA. Hospital admissions that began in the emergency department, 2003. Rockville, MD: Agency for Healthcare Research and Quality; 2006 (Statistical Brief; vol 1).

3. Schafermeyer RW, Asplin BR. Hospital and emergency department crowding in the United States. Emerg Med (Fremantle). 2003;15:22-7.

4. American Hospital Association. Emergency departments: An essential access point to care. Trendwatch. 2001;3:1-8.

5. Lewin Group. Emergency department overload: A growing crisis: The results of the AHA survey of emergency department (Ed) and hospital capacity. Falls Church, VA: American Hospital Association; 2002.

6. Brewster LR, Rudell LS, Lesser CS. Emergency room diversions: A symptom of hospitals under stress. Issue Brief Cent Stud Health Syst Change. 2001(38):1-4.

7. US General Accounting Office. Hospital emergency departments: Crowding conditions vary among hospitals and communities. Washington, DC: US General Accounting Office; 2003 [citado 15 Sep 2009]. Disponible en: URL: http://www.gao.gov/ new. items/d03460.pdf.

8. Lerner EB, Moscati RM. The golden hour: Scientific fact or medical "urban legend"?. Acad Emerg Med. 2001;8: 758-760.

9. Committee on the future of emergency care in the US hospital based emergency care at the breaking point. Washington, DC: National Academy Press; 2007 [citado 15 Sep 2009] Disponible en: URL: http://www.nap.edu/catalog.php?record_id=11621.

10. Committee on the Future of Emergency Care in the US Emergency Medical Services at the Crossroads. Washington,
DC: National Academy Press; 2007 [citado 15 Sep 2009]. Disponible en: URL: http: //www.iom.edu/?id=48898.

11. Committee on the Future of Emergency Care in the US Emergency Care for Children: Growing pains. Washington, DC: National Academy Press; 2007 [citado 15 Sep 2009]. Disponible en: URL: http://www.nap.edu/catalog.php?record_id=11655.

12. González López-Valcarel, B, Barber Pérez P, Oferta y necesidad de médicos especialistas en España (2006-2030). Madrid: Ministerio de Sanidad y Consumo de España; 2007 [citado 15 Sep 2009]. Disponible en: URL: www.msc.es/novedades/docs/ necesidadesEspeciales06_30.pdf.

13. Ministerio de Sanidad y Consumo de España. Oferta y demanda de especialistas 2008-2025. Madrid: Ministerio de Sanidad y Consumo de España; 2009 [citado 15 Sep 2009]. Disponible en: URL: http://www.la-moncloa.es/NR/rdonlyres/E87EAAC5EOEB-4CDA-8521.

14. Ribo M, Molina CA, Pedragosa A, Sanclemente C, Santamarina E, Rubiera $M$, et al. Geographic differences in acute stroke care in Catalunya: Impact of a regional interhospital network. Cerebrovasc Dis. 2008;26:284-8.

15. Di Bartolomeo S, Valent F, Rossi C, Beltrame F, Anghileri A, Barbone F. Geographical differences in mortality of severely injured patients in Italy. Eur J Epidemiol. 2008;23:289-94.

16. Rothen HU, Stricker K, Einfalt J, Bauer P, Metnitz PG, Moreno RP, et al. Variability in outcome and resource use in intensive care units. Intensive Care Med. 2007;33:1329-36.

17. Roberts CM, Barnes S, Lowe D, Pearson MG. Evidence for a link between mortality in acute COPD and hospital type and resources. Thorax. 2003;58:947-9.

18. Angrisano C, Farrell D, Kocher B, Laboissiere M, Parker S. Accounting for the cost in the United States. Sao Paulo, Brazil: McKinsey and Co.; 2007 [citado 15 Sep 2009]. Disponible en: URL: http://www.mckinsey.com/mgi/rp/healthcare/accounting_ cost_healthcare.asp.

19. California Health Care Foundation. Snapshot: Health care costs 101. Oakland, CA: California Health Care Foundation; 2005 [citado 15 Sep 2009]. Disponible en: URL: http://www.chcf. org/documents/insurance/HCCosts10105.pdf.

20. Wagner DP, Wineland TD, Knaus WA. The hidden costs of treating severely ill patients: Charges and resource consumption in an intensive care unit. Health Care Financ Rev. 1983;5:81-6.

21. Noseworthy TW, Konopad E, Shustack A, Johnston R, Grace M. Cost accounting of adult intensive care: Methods and human and capital inputs. Crit Care Med. 1996;24:1168-72.

22. Roca J, Pérez J, Colmenero M, Muñoz H, Alarcón L, Vázquez G. Competencias profesionales para la atención al paciente critico. Más allá de las especialidades. Med Intensiva. 2007;31:473-84.

23. Thibault GE, Mulley AG, Barnett GO, Goldstein RL, Reder VA, Sherman EL, et al. Medical intensive care: Indications, interventions, and outcomes. N Engl J Med. 1980;302:938-42.

24. Kestin IG, Miller BR, Lockhart CH. Auditory alarms during anesthesia monitoring. Anesthesiology. 1988;69:106-9.

25. Joint Commission on Accreditation of Health Care Organizations. Sentinel event alert. Preventing ventilator-related deaths and injuries. Washington, DC: Joint Commission; 2002. p. 25. [citado 15 Sep 2009]. Disponible en: URL: http://www.jointcommission. org/SentinelEvents/SentinelEventAlert/sea_25.htm.

26. Koski EM, Makivirta A, Sukuvaara T, Kari A. Clinicians' opinions on alarm limits and urgency of therapeutic responses. Int J Clin Monit Comput. 1995;12:85-8.

27. Balogh D, Kittinger E, Benzer A, Hackl JM. Noise in the ICU. Intensive Care Med. 1993;19:343-6.

28. Aaron JN, Carlisle CC, Carskadon MA, Meyer TJ, Hill NS, Millman RP. Environmental noise as a cause of sleep disruption in an intermediate respiratory care unit. Sleep. 1996;19: 707-710. 
29. Topf $M$, Dillon $E$. Noise-induced stress as a predictor of burnout in critical care nurses. Heart Lung. 1988;17:567-74.

30. Quesada Suescun A, Burón Mediavilla F, Castellanos Ortega A, Vicente-Mazariegos I, González Fernández C, Olalla Antolkn JJ, et al. Formación en la asistencia al paciente crítico y politraumatizado: papel de la simulación clínica. Med Intensiva. 2007;31:187-93.

31. Vázquez G, Roca J, Blanch L. El reto de la web 2.0 «UCI Virtual». Med Intensiva. 2009;33:84-7.

32. Martin M, Cabré L, Ruiz J, Blanch LI, Blanco J, Castillo F, et al. Indicadores de calidad en el enfermo crítico. Med Intensiva. 2008;32:23-32.
33. Gutiérrez Cia I, Obon Azuara B, Aibar Remon C. Análisis de los «casi errores». Un nuevo enfoque en el estudio de los acontecimientos adversos. Med Intensiva. 2008;32: 143-146.

34. Abizanda Campos R, Ferrandiz Selles A, Reig Valero R. PDMS o UDMS. Dos perspectivas de gestión clínica en Medicina Intensiva. Med Intensiva. 2008;32:354-60.

35. Maná F, Sellés J, Bertolín R. Tendencias futuras de conectividad en entornos fijos, nómadas y móviles. Madrid, España: Fundación OPTI; 2007 [citado 8 Ene 2009]. Disponible en: URL. http://www.opti.org/publicaciones/pdf/resumen24. pdf. 